\title{
gु Direct relations between morphology and transport in Boolean models
}

\author{
Christian Scholz, ${ }^{1,2}$ Frank Wirner, ${ }^{1}$ Michael A. Klatt, ${ }^{3}$ Daniel Hirneise, ${ }^{1}$ Gerd E. Schröder-Turk, ${ }^{4,3}$ \\ Klaus Mecke, ${ }^{3}$ and Clemens Bechinger ${ }^{1,5}$ \\ 12. Physikalisches Institut, Universität Stuttgart, Pfaffenwaldring 57, 70569 Stuttgart, Germany \\ ${ }^{2}$ Institut für Multiskalensimulation, Nägelsbachstraße 49b, Friedrich-Alexander Universität Erlangen-Nürnberg, 91052 Erlangen, Germany \\ ${ }^{3}$ Institut für Theoretische Physik, Friedrich-Alexander Universität Erlangen-Nürnberg, Staudtstraße 7B, 91058 Erlangen, Germany \\ ${ }^{4}$ Murdoch University, School of Engineering \& IT, Maths \& Stats, 90 South Str., Murdoch WA 6150, Australia \\ ${ }^{5}$ Max-Planck-Institut für Intelligente Systeme, Heisenbergstraße 3, 70569 Stuttgart, Germany
}

(Received 22 July 2015; published 30 October 2015)

\begin{abstract}
We study the relation of permeability and morphology for porous structures composed of randomly placed overlapping circular or elliptical grains, so-called Boolean models. Microfluidic experiments and lattice Boltzmann simulations allow us to evaluate a power-law relation between the Euler characteristic of the conducting phase and its permeability. Moreover, this relation is so far only directly applicable to structures composed of overlapping grains where the grain density is known a priori. We develop a generalization to arbitrary structures modeled by Boolean models and characterized by Minkowski functionals. This generalization works well for the permeability of the void phase in systems with overlapping grains, but systematic deviations are found if the grain phase is transporting the fluid. In the latter case our analysis reveals a significant dependence on the spatial discretization of the porous structure, in particular the occurrence of single isolated pixels. To link the results to percolation theory we performed Monte Carlo simulations of the Euler characteristic of the open cluster, which reveals different regimes of applicability for our permeability-morphology relations close to and far away from the percolation threshold.
\end{abstract}

DOI: 10.1103/PhysRevE.92.043023

PACS number(s): 47.56.+r, 61.43.Gt, 47.61.-k

\section{INTRODUCTION}

The flow of liquids through porous media is of considerable importance in many scientific areas, such as groundwater pollution, secondary oil recovery, or blood perfusion inside the human body [1,2]. Although the literature on porous media has been growing rapidly over the last decades, it is still not fully understood how transport properties of liquids through porous materials can be related to the microstructure even for single-phase flow.

Analytical results exist for regular structures [3], and rigorous bounds have been proposed for random media [4]. Yet, it is still an open question, in particular for many random systems, which structural properties determine the permeability, i.e., the ability of a material to conduct fluid flow.

The permeability $k$ of a porous medium, which is perhaps the most fundamental flow property, relates the flow rate $Q$ and the applied pressure $P$, according to Darcy's law

$$
Q=-\frac{k A}{\eta} \nabla P,
$$

where $A$ is the cross-sectional area of the material and $\eta$ the viscosity of the fluid. In Eq. (1) one assumes a linear relation between flow and pressure. This is strictly speaking only valid for low Reynolds number flow $(\operatorname{Re}<10)$ of viscous liquids, where Darcy's law can be derived from the Stokes equation. However, the determination of $k$ still requires the solution of the flow problem on the microscale, which for many random systems is a huge computationally expensive task.

For this reason preferably simple relations between geometry and flow in porous media are typically used to predict the permeability. One approach is based on a morphological reconstruction of disordered structures with the help of
Boolean models, i.e., structures formed by randomly placed and oriented grains with suitably chosen parameters, which was introduced in Refs. [5,6]. It has been shown that if a set of morphological quantifiers of the pore space, so-called Minkowski functionals (MFs), between the original and a reconstructed structure agrees, also the conductivity and elasticity of both materials are similar. Therefore Boolean models are, despite their simplicity, applicable to morphologically describe properties of random porous media, even if the formation process of the original structure might be more complicated. Additionally, the question arises whether dynamic quantities, such as the permeability, can be directly deduced from the MFs. Such a relation has been proposed in Ref. [7], where $k$ has been related to one of the MFs, viz., the Euler characteristic of the conducting cluster in Boolean models of overlapping circles and ellipses. However, this relation requires prior knowledge of the grain density, which might not be available for arbitrary structures. Additionally, other quantities, such as the surface area might influence $k[8,9]$.

In this paper we discuss a generalization to arbitrary microstructures and the range of validity close to and far away from the percolation threshold, which is the highest porosity below which there is no sample-spanning conducting phase. A definition of the effective grain density based on reconstruction schemes via Boolean models and Minkowski functionals, allows us to apply the relation to both phases of Boolean models, i.e., void and grain percolation. However, deviations are found, which we attribute to the occurrence or disappearance of individual occupied lattice sites, which have a significant effect on the topology and, thus, on the effective grain density, without strongly affecting the fluid flow. We find that in the low grain-density limit the Euler characteristic's dependence on the porosity is equivalent to the effective medium result for the conductivity. Furthermore, numerical results enable us 
to the determine the range of validity close to the percolation threshold and to link our results to percolation theory.

The paper is organized as follows. In Sec. II, we discuss semiempirical relations between geometric and transport properties from the established literature. In Sec. III, we explain our microfluidic experiments, which allow us to determine flow properties of quasi-two-dimensional representations of Boolean models. The details of our model structures are explained in Sec. IV and the corresponding Minkowski functionals are discussed in Sec. V. The numerical results of the permeability and conductivity, in particular the validity of the Katz-Thompson model, are addressed in Sec. VI. In Sec. VII, we evaluate our experimental results for void percolation and introduce a power-law relation between Euler characteristic and permeability. We evaluate the possible extension to arbitrary structures, by defining an effective grain number and check its validity for grain percolation. Analytical results for low grain density and computational results close to the percolation threshold are presented in Secs. VIII and IX, which allows us to establish the valid range of our assumption. Finally a summary of the results is presented in Sec. X.

\section{SEMIEMPIRICAL RELATIONS}

Many semiempirical relations for pressure-driven single phase flow based on the results for flow through regular sphere packings have been proposed $[2,10]$. For random porous media, Katz and Thompson proposed a relation between $k$ and the electrical conductivity $\sigma$ of the fluid-saturated phase:

$$
k=c l_{c}{ }^{2} \frac{\sigma}{\sigma_{0}}
$$

where $c$ is a constant that depends on the local pore morphology, $\sigma_{0}$ is the bulk conductivity of the fluid and $l_{c}$ is a critical pore diameter, equal to the size of the bottle neck of the conducting phase, i.e., the diameter of the largest hardsphere which can freely penetrate the porous material [11]. Equation (2) can be derived by using ideas from percolation theory to treat the conductance problem through a porous structure as a percolation problem with a critical threshold value for the conductance and assuming the same critical exponents for the conductance and flow problem. Of course this approach depends on the universality of critical exponents, which might be different in two and three dimensions and depend, for example, on the pore size distribution [12-14]. Yet, the validity of Eq. (2) has been shown for various types of natural rock and random media [15-18].

As Eq. (2) relates conductivity and permeability, it is typically called a cross-property relation to distinguish it from a purely geometrical relation to transport properties. The ratio of the bulk conductivity of the fluid $\sigma_{0}$ to the effective conductivity of the porous medium saturated with that fluid $\sigma$ is called formation factor

$$
F=\frac{\sigma_{0}}{\sigma}
$$

For many random porous media it is assumed that $F$ is related to the porosity $\phi$, i.e., the volume fraction of the conducting phase, by Archie's law

$$
\frac{\sigma}{\sigma_{0}}=\left(\frac{\phi-\phi_{c}}{1-\phi_{c}}\right)^{\mu}
$$

where $\phi_{c}$ is the percolation threshold [19,20], i.e., the porosity at which the type of porous material ceases to percolate and $\mu$ is an exponent, which typically depends on the porosity and morphology, but might be associated with the critical conductivity exponent close to $\phi_{c}$, where it is expected to be universal and $\mu \approx 1.3$ in two dimensions [12,21-24].

Two issues limit the applicability of Eq. (4): (i) according to percolation theory, Eq. (4) is only valid close to $\phi_{c}$ and (ii) $\phi_{c}$ is a parameter, which explicitly depends on the formation process of a structure and cannot be deduced from single finite realizations of this process [25,26]. Astonishingly, even for simple formation processes, such as percolation on a lattice or continuum percolation of overlapping grains, $\phi_{c}$ is not known analytically. To resolve these two issues, a relation independent of $\phi_{c}$ is required to accurately predict $\sigma$ and $k$ of arbitrary finite-sized porous media.

\section{EXPERIMENTAL METHODS}

We create micromodel structures for the experimental determination of the permeability using the well-established soft-lithography method [27,28]. The samples consist of three separated rectangular channels of height $h$ on the order of $10 \mu \mathrm{m}$, which are connected to pressure reservoirs [see Figs. 1(a) and 1(b)]. Each channel has a length on the order of $10 \mathrm{~mm}$ and a width of $3.5 \mathrm{~mm}$. A porous structure of length $L_{\text {struct }}=3.5 \mathrm{~mm}$ is placed in the center of the middle channel. The two outer channels contain no porous structures, except for a diluted array of supporting pillars of diameter $10 \mu \mathrm{m}$ with typical spacing of $500 \mu \mathrm{m}(\phi>0.999)$. A dilute suspension of colloidal particles of diameter $\sigma=3 \mu \mathrm{m}$ is injected into this microfluidic device as tracer particles. For macroscopic experiments the permeability according to Eq. (1) is typically determined by applying a fixed flow rate and then measuring the pressure drop across the porous structure. However, for soft-lithographic channels to prevent feedback and leakage, only small flow rates must be applied, which requires high-sensitivity motorized syringes. Additionally, an accurate determination of the pressure drop between inlet and outlet is required, which is difficult to achieve with macroscopic pressure transducers.

For this reason we use particle tracking velocimetry [29-31] to determine the average velocity of the injected colloidal tracer particles as a function of the applied pressure $\Delta P$ in each channel. Here, in contrast to the fixed flow-rate method, the applied hydrostatic pressure can be tuned accurately by varying the water level in the two reservoirs. The outer channels act as references to calibrate the relationship between particle velocity and fluid velocity. As shown in previous experiments [32-34], the average particle speed within thin rectangular channels typically deviates significantly from the average fluid velocity, depending on the particle diameter, the height of the channel and the particles' gravitational height $[35,36]$. However, the mean fluid velocity $\bar{v}$ and mean particle 
(a)

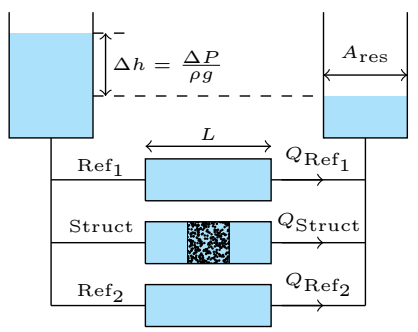

(b)

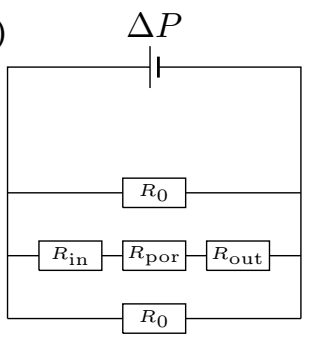

(c)

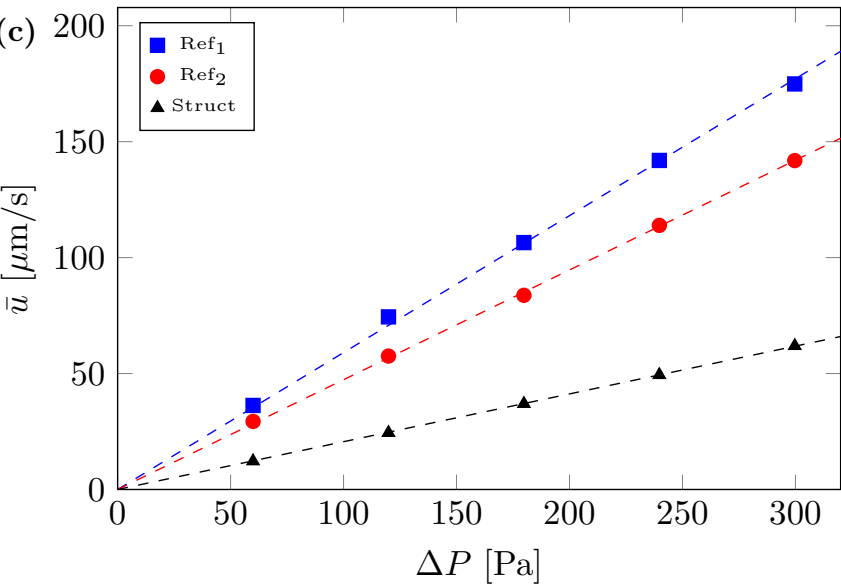

FIG. 1. (Color online) Summary of experimental method: (a) A microfluidic sample with a porous structure in the center of the middle channel is created. A dilute suspension of colloidal particles is injected into the device and an external hydrostatic pressure is applied with the help of two reservoirs. The flow rate through the channel is measured from the velocity of the colloidal tracers. Unpatterned reference channels for calibration are added parallel to the structured channel. To account for possible deviations in the channel height two reference channels are used to cross check the results. (b) Equivalent circuit diagram of hydraulic resistances $R_{\mathrm{hyd}}=\frac{\Delta P}{Q}=\frac{\eta L}{k A}$. (c) Mean particle velocity $\bar{u}$ measured in the two reference and the middle channel containing the porous structure as a function of the applied pressure. The dashed lines are fits to the data points. The average particle velocity $\bar{u}$ is lower in the structured channel due to the lower permeability of the porous part. The fluid velocity in the structure channel is noticeably slower than in the reference channels. Similar fluid velocities are found in the reference channels, however, differences are caused by different length $(\Delta L \approx 500 \mu \mathrm{m})$ and height $(\Delta h \approx 0.5 \mu \mathrm{m})$ of the reference channels and must be taken into account. The error bars are smaller than the symbol sizes.

velocity $\bar{u}$ in a channel are proportional [37], so that

$$
\bar{v}=c_{d} \bar{u} .
$$

The fluid flow problem in the reference channels, which are assumed to be infinite parallel plates, can be solved analytically for a given pressure drop $\Delta P$. The permeability $k_{0}$ of the reference channels is equal to $h^{2} / 12$ [38]. Therefore, the calibration factor $c_{d}$ can be obtained by calculating the theoretical value for $\bar{v}$ with Eq. (1) and measuring $\bar{u}$. Since $k_{0} \propto h^{2}$, the calibration is very sensitive to the height of the structure (and its spatial variations) [38]. We double check the calibration for each sample with two reference channels to account for differences between individual samples, which can occur during the lithographic process. Figure 1(c) shows an exemplary plot of $\bar{u}$ vs. $\Delta P$ for the three channels of one sample. As illustrated, the fluid velocity in the channel containing the porous structure is considerably slower, while in the reference channels it is similar [small differences are caused by the different length $(\Delta L \approx 500 \mu \mathrm{m})$ and slightly different height of the channels $(\Delta h \approx 0.5 \mu \mathrm{m})$, which result from the production process].

By considering the middle channel as a series of hydraulic resistances $R_{\mathrm{hyd}}=\frac{\Delta P}{Q}$, as depicted in the equivalent circuit diagram in Fig. 1(b), we can determine the permeability of the central structure directly from the flow rate. If all flow rates are known, the relative permeability (normalized to the $k_{0}$ of the reference channel) of a structure is given by

$$
\frac{k_{\text {struct }}}{k_{0}}=\frac{L_{\text {struct }}}{L_{\text {ref }} \frac{Q_{\text {ref }}}{Q_{\text {struct }}}-\left(L_{\text {in }}+L_{\text {out }}\right)},
$$

where $L_{\text {ref }}$ is the total length of the reference channel and $L_{\text {in/out }}$ are the length of the inlet and outlet of the structure channel [see Fig. 1(a)].

From Eq. (5) we have $Q_{\text {ref/struct }}=c_{d} \times \bar{u}_{\text {ref/struct }} \times A$, which allows us to directly determine the permeability from a parallel measurement of $\bar{u}$ in the reference and structure channels. It is important to note, that crossover effects between the different sections of the middle channel are neglected here, i.e., the individual segments are assumed to be well connected.

\section{BOOLEAN MODELS}

Boolean models are well established models for porous materials from stochastic geometry [39-41]. There, porous structures are composed of overlapping grains with random position and orientation (i.e., points in a plane are chosen randomly in a Poisson point process). At each point a grain is placed and in the case of anisotropic grains orientations are also chosen randomly from a uniform distribution. In this article, we consider models of randomly overlapping monodisperse circles (ROMCs) and randomly overlapping monodisperse ellipses (ROMEs) with isotropic random orientation. We also simulate systems of overlapping monodisperse rectangles (ROMRs) with random orientation, which allows us to minimize discretization errors, because such grains can be, in contrast to spheres, directly represented by polygons of arbitrary precision. Each structure is parametrized by the type, aspect ratio and number $N$ or number density $\rho=N / L^{2}$ of grains, where $L$ is the linear system size. Examples of these models are shown in Fig. 2 for grain percolation [Figs. 2(a)2(c)] and void percolation [Figs. 2(d)-2(e)]. The white phase corresponds to the conducting phase. Exchanging the two phases results in totally different pore space morphologies.

For the experimental and numerical determination of conductivity and permeability, we create five realizations of ROMC and ROME structures on a quadratic two-dimensional (2D) lattice with linear size $L=4000$ in pairs of equal open porosity $\phi_{o}$, i.e., the volume fraction of only the samplespanning part of the conducting phase. The circles have a radius of $r=34$ in units of lattice sites and the ellipses have a long and short semiaxis of $a=96$ and $b=12$. In the microfluidic samples this equals $r \simeq 30 \mu \mathrm{m}$ and $a \simeq 84 \mu \mathrm{m}$. The morphological properties of the resulting structures and 


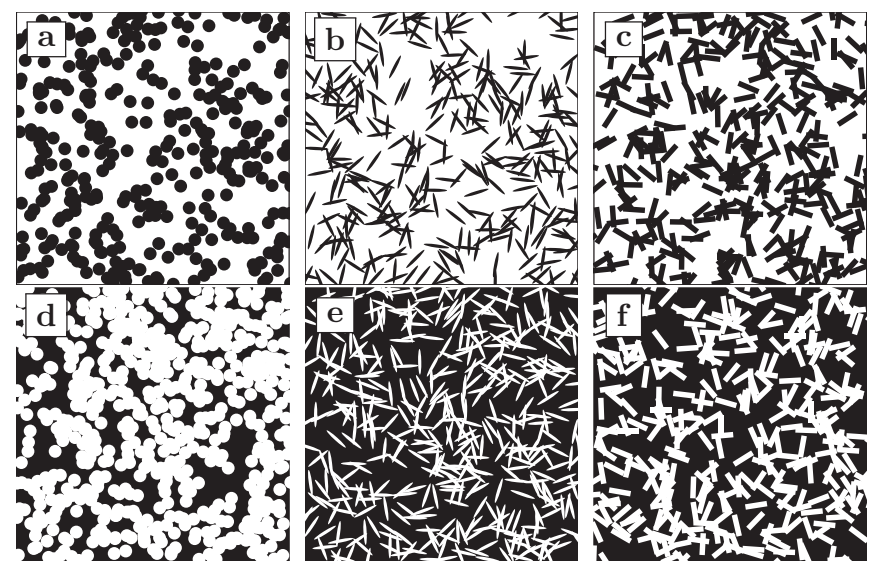

FIG. 2. Boolean models of overlapping grains for (a) circles, (b) ellipses, and (c) rectangles. A set of $N$ points is selected randomly in a plane (total area $L^{2}$ ) (Poisson process). At each point a grain with random orientation is placed. Each structure is characterized by its point density $N / L^{2}$. The white phase corresponds to the conducting phase. (a)-(c) are structures, which we classify as void percolation, whereas the structures (d)-(f) exhibit grain percolation.

their percolating phase (marked with an index $o$ for open) are summarized in Table I.

\section{MINKOWSKI FUNCTIONALS}

Minkowski functionals are morphological measures, corresponding to volume and surface integrals of geometric sets, which are particularly useful for characterizing random structures [39,42-47]. In two dimensions the Minkowski functionals of a compact set $\mathfrak{A}$ are given by

$$
\begin{gathered}
W_{0}(\mathfrak{A})=\int_{\mathfrak{A}} d^{2} \boldsymbol{r}, \\
W_{1}(\mathfrak{A})=\frac{1}{2} \int_{\partial \mathfrak{A}} d \boldsymbol{r}, \\
W_{2}(\mathfrak{A})=\frac{1}{2} \int_{\partial \mathfrak{A}} \frac{1}{R} d \boldsymbol{r},
\end{gathered}
$$

where $R$ is the radius of curvature. From this definition, the MF in the continuum can be identified with area $V$, perimeter $P$, and Euler characteristic $\chi$ of a set: $V=W_{0}, P=2 W_{1}$, and $\chi=W_{2} / \pi$, so that the values for a unit disk are $W_{i}=\pi$. On a $2 \mathrm{D}$ lattice the normalization of $W_{i}$ is chosen differently, so that the values for a unit pixel are $W_{i}=1$, i.e., $V=W_{0}, P=4 W_{1}$, and $\chi=W_{2}$. A schematic illustration of the MF is given in Fig. 3.

The Euler characteristic is a topological constant, which in two dimensions is equivalent to the number difference between connected components and holes in a set. This quantity is particularly useful for the characterization of percolating structures, because for many random sets, $\chi$ becomes zero close to the percolation threshold, i.e., the number of connected components of both phases are approximately the same [42].

For Boolean models in the continuum the MFs of individual grains (local MFs $w_{i}$ ) and the mean MFs of realizations of the model (global MFs $W_{i}$ ) with mean density $\rho=\langle N\rangle / L^{2}$ are related by
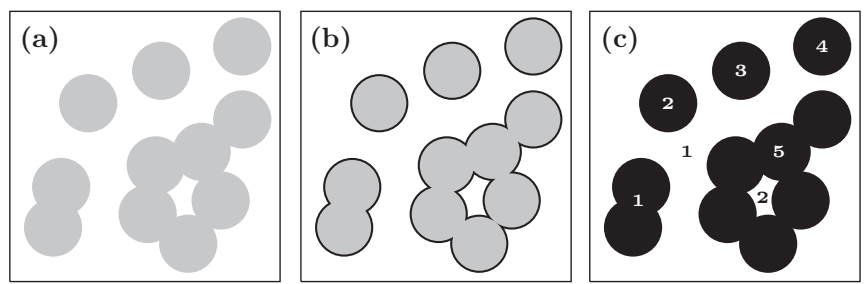

FIG. 3. Schematic illustration of the Minkowski functionals: (a) The area $V$ of the conducting phase is shown in white, (b) the perimeter $P$ corresponds to the length of the black boundary, and (c) the Euler characteristic $\chi$ is the number difference of the connected components of each phase, which in the case shown would be $2-5=-3$. The open Euler characteristic $\chi_{o}$ (similar to open porosity $\phi_{o}$ and open perimeter $S_{o}$ ) does not count any inclusions, i.e., $\chi_{o}=1-5=-4$, as the inclusion in cluster No. 5 would be neglected.

$$
\begin{gathered}
W_{0}(\rho) / L^{2}=1-e^{-\rho w_{0}}, \\
W_{1}(\rho) / L^{2}=\rho w_{1} e^{-\rho w_{0}}, \\
W_{2}(\rho) / L^{2}=\rho\left(w_{2}-\frac{\left(2 w_{1}\right)^{2}}{4} \rho\right) e^{-\rho w_{0}} .
\end{gathered}
$$

However, porous structures, in particular when obtained from experimental data, are often represented as discretized binary data sets on a lattice. For such data it is convenient to define the MFs in a discrete system. For Boolean models on a lattice with eight-point connectivity (horizontal, vertical, and diagonal neighbors) the relations are

$$
\begin{gathered}
W_{0}(\rho) / L^{2}=1-e^{-\rho w_{0}}, \\
W_{1}(\rho) / L^{2}=e^{-\rho w_{0}}\left(1-e^{-\rho w_{1}}\right), \\
W_{2}(\rho) / L^{2}=e^{-\rho w_{0}}\left(-1+2 e^{-\rho w_{1}}-e^{-\rho\left(2 w_{1}+w_{2}\right)}\right) .
\end{gathered}
$$

In both cases these equations are invertible. Such an inversion has been used to determine Boolean models with grain compositions with matching $w_{i}$ to reconstruct natural porous media, such as Fontainebleau sandstone [5].

Numerically the Minkowski functionals can also be calculated for the percolating (open) phase. From this we obtain $\phi_{o}$, $S_{o}$, and $\chi_{o}$.

\section{KATZ-THOMPSON MODEL}

In the literature it is controversially discussed whether permeability and conductivity have different or equal scaling exponents [2]. In the Katz-Thompson model equal scaling exponents are assumed, which gives a relationship between conductivity and permeability based on arguments from percolation theory. This equality is relevant in our case, since many analytical results on the pore scale are only obtained for the conductivity, but not the permeability of porous media.

The length scale that determines the permeability in the Katz-Thompson model is identified as the critical pore diameter [see Fig. 4(b)]. Due to the quasi-2D geometry of our 


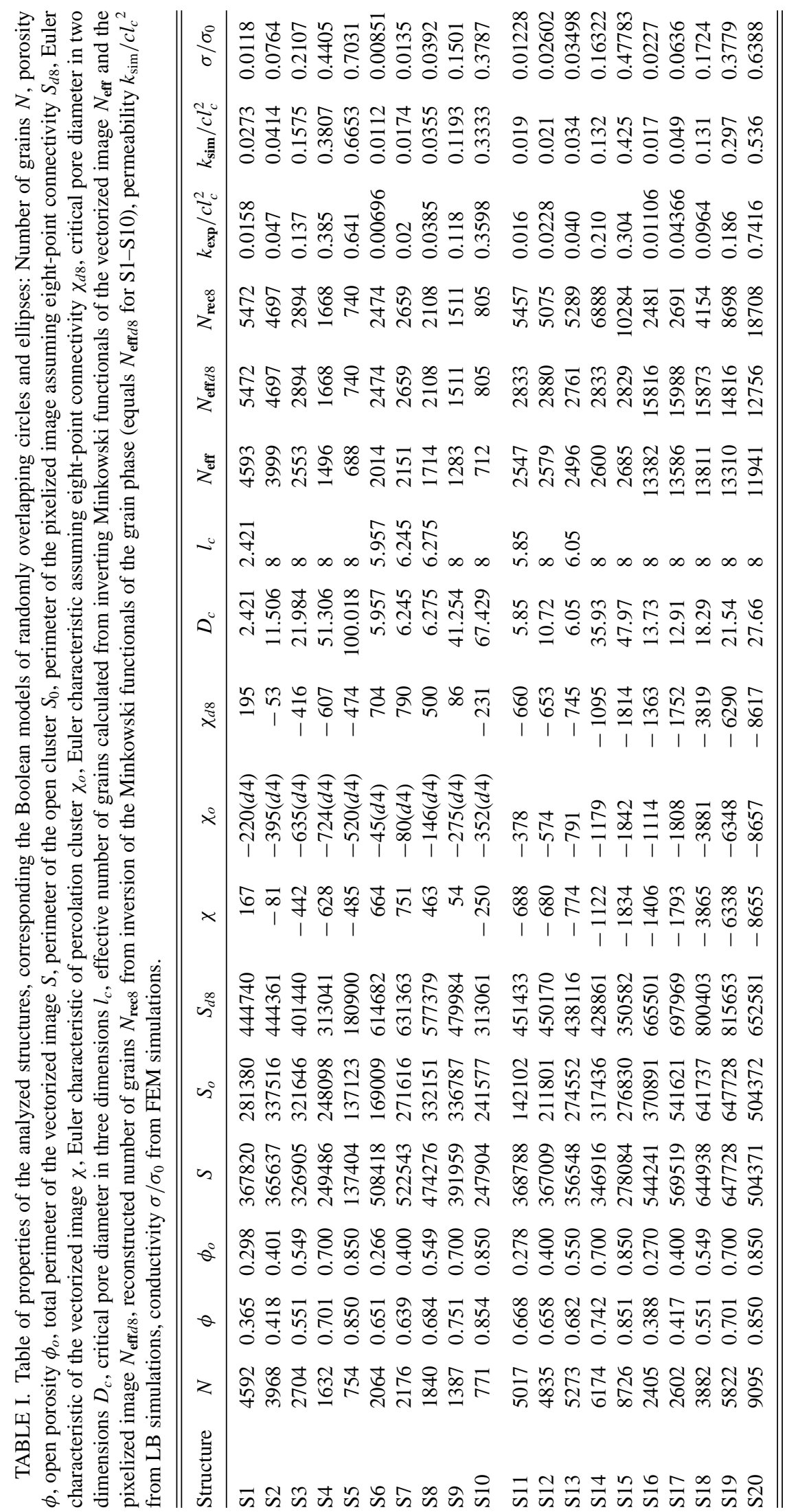




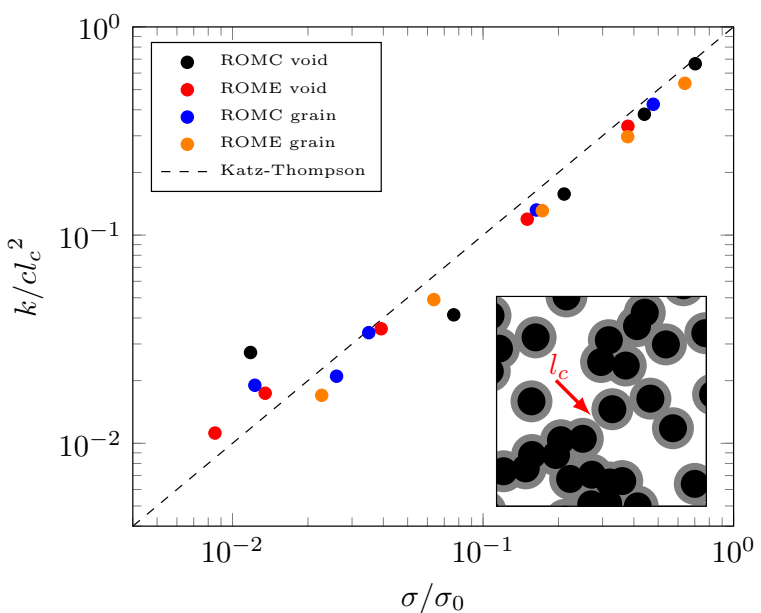

FIG. 4. (Color online) Cross-property relation between permeability $k$ determined by LB simulations and conductivity $\sigma$ obtained from FEM simulations. The conductivity is normalized by the bulk conductivity $\sigma_{0}$. The permeability is normalized by the square of the critical pore diameter $l_{c}$ and a constant $c=1 / 12$, determined from the limit $l_{c} \rightarrow h$ and $\phi \rightarrow 1$, where the system equals the flow problem between infinitely large parallel plates with spacing $l_{c}$. Inset: Determination of the critical pore diameter $l_{c}$ from parallel surfaces.

samples this critical diameter is given by

$$
l_{c}=\min \left(D_{c}, h\right),
$$

where $D_{c}$ is the actual 2D critical diameter. If this diameter is greater than the height $h$ of the structure, $h$ confines the flow and becomes the relevant length scale. For our structures we determined $l_{c}$ directly from the images of the structures computing the Euclidean distance transform (EDT). For each point in the conducting phase of the sample the EDT assigns the distance to the closest point on the surface. From this $l_{c}$ can be easily identified [48,49] (see inset of Fig. 4). The constant $c=1 / 12$ is chosen to fit the dilute grain limit, where $c l_{c}^{2}=h^{2} / 12$.

In Fig. 4 the relative permeability is shown in dependence of the conductivity, both determined numerically from lattice-Boltzmann (LB) and finite-element (FEM) simulations, respectively. The prediction of the Katz-Thompson model is depicted as a dashed line. In particular for larger $\phi$, the data agrees very well with the Katz-Thompson model, with only a slight deviation of the permeability towards lower values than predicted. Additionally even for low permeabilities the predictions deviate by less than a factor of two for all but one structure.

For $\phi \rightarrow 1$, this agreement is not surprising, since the height of the structure is much smaller than the distance between the obstacles, which leads to an equivalence of the flow and the conductance problem since the weight of the different pathways is the same for flow and conductance. As the flow profile is locally equivalent to flow confined between infinitely large parallel plates the system can be thought of as a network of hydraulic conductors with conductivity proportional to $h^{3}$. In both cases, a homogeneous current or flow is only disturbed by isolated obstacles, which only locally influences the (hydraulic) conductance of the porous structure, without changing the hydraulic radius, which remains on the order of the height $h$.

However, this is not the case close to the percolation threshold $\phi_{c}$. Katz and Thompson argue that $\sigma$ and $k$ follow similar universal power laws close to the critical porosity with an accurate choice of the critical pore diameter. According to
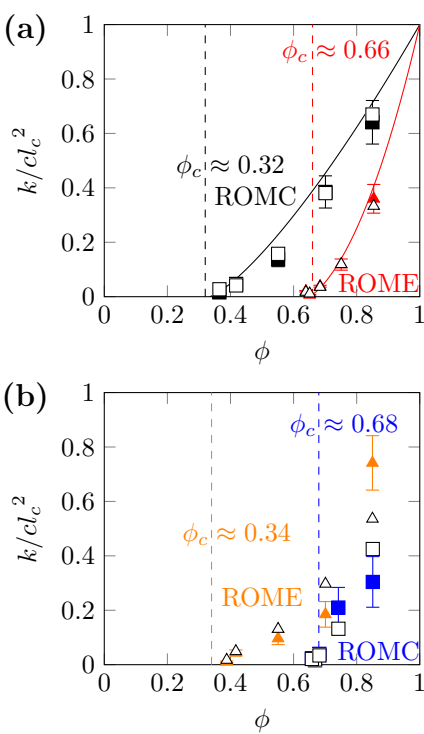
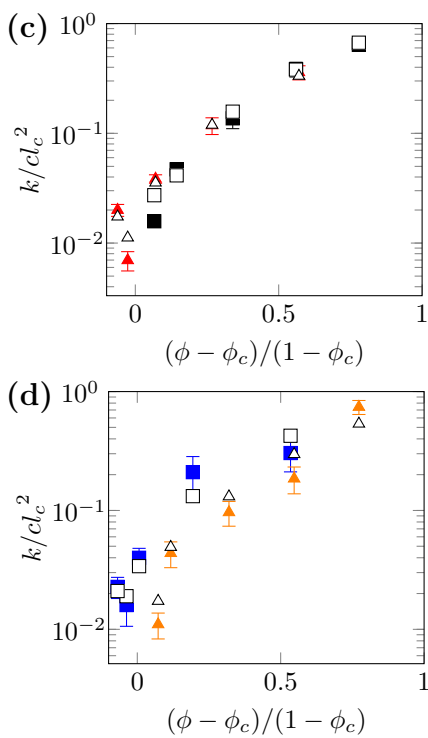
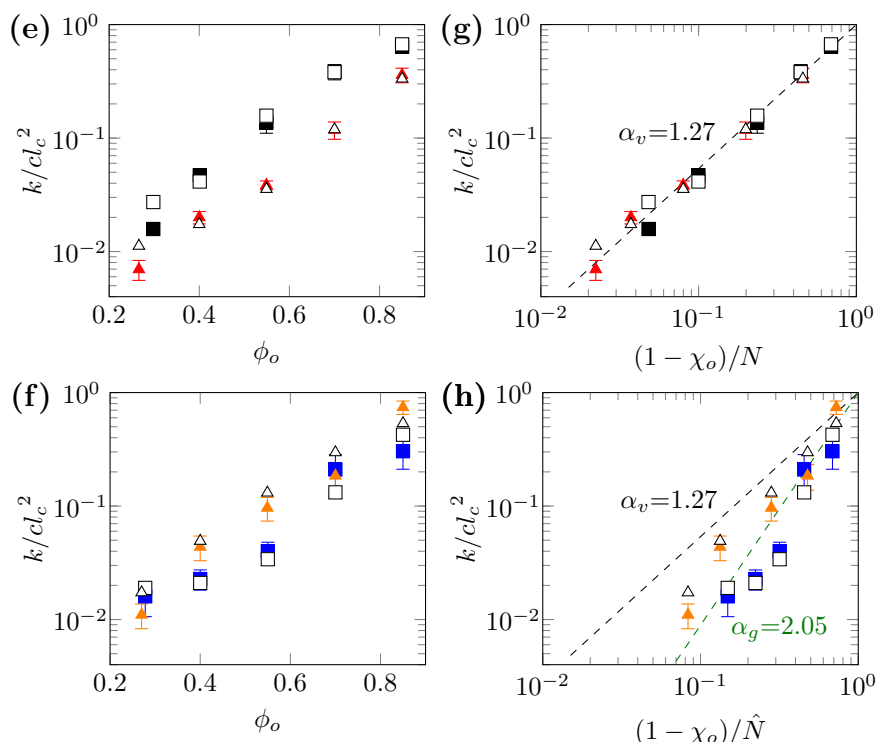

FIG. 5. (Color online) Experimentally (closed symbols) and numerically (open symbols) determined permeability $k$ of void (top) and grain percolation (bottom) vs. different morphological properties: (a), (b) porosity $\phi$, as expected $k / c l_{c}^{2}$ vanishes around $\phi_{c}$ and goes to 1 for $\phi \rightarrow 1$; (c), (d) rescaled porosity, data points collapse with some deviations due to finite-size effects; (e), (f) open porosity, for void percolation ROMC structures have higher permeabilities, for grain percolation ROME structures have higher permeability at equal $\phi_{o}$; and (g), (h) Euler characteristic: data collapses onto a single curve for void percolation, but for grain percolation deviations are found. The dashed lines in ( $\mathrm{g}$ ) and (h) are fits to Eq. (17) with one free parameter $\alpha$. Error bars are only shown if larger than symbol size. 

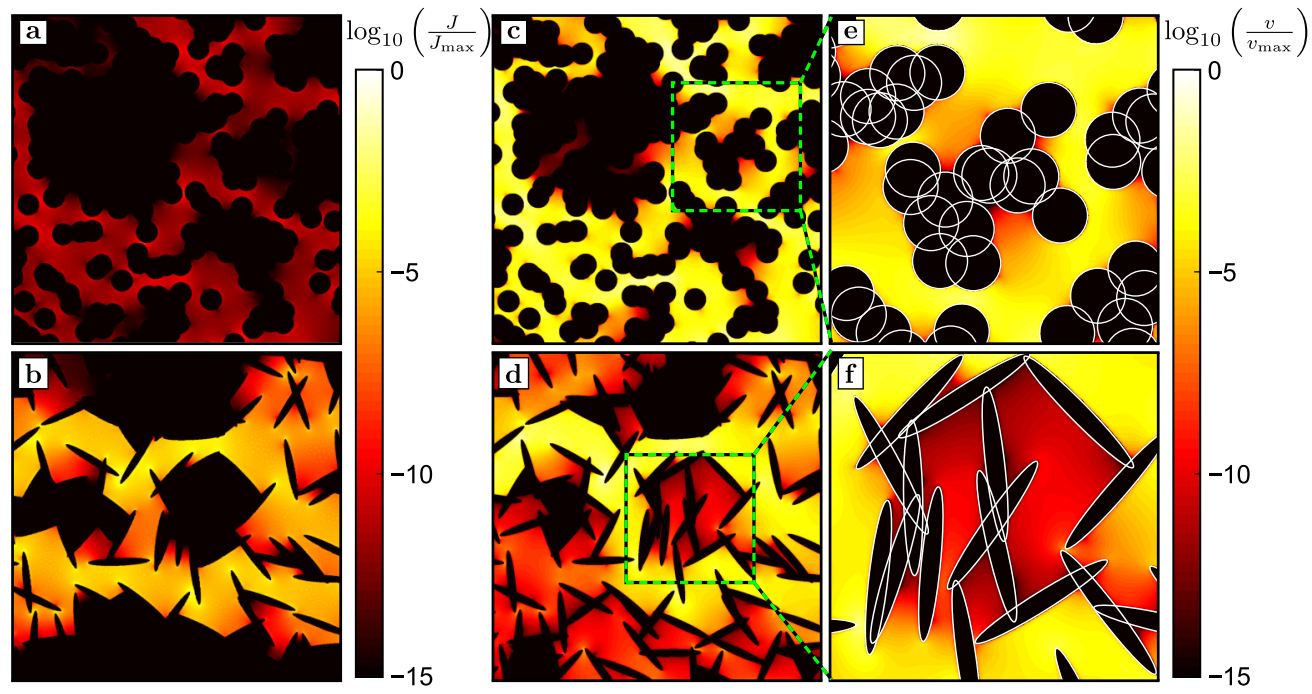

FIG. 6. (Color online) (a), (b) Current density magnitude from finite-element simulations of the Laplace equation (i.e., conductivity) normalized to the total maximum current. (c), (d) Fluid velocity magnitude from lattice-Boltzmann simulations. For elliptical grains the overlap leads to pronounced stagnant parts, while circular grains form more compact obstacles, as illustrated in (e), (f) respectively. Within such stagnant parts a strong decrease of both the current and the flow velocity is observed. However the decrease of $j$ appears to be faster, as observed in Ref. [50].

some authors this is only true for two dimensions [12-14]. Otherwise, nonuniversal power-law exponents have to be considered. To obtain further insight into this problem we show the current density and the flow velocity magnitude for representative ROMC and ROME structures in Fig. 6. Even though the fields share some morphological features, such as the principal flow paths, the decay of the current magnitude into dead ends appears to be faster than that of the fluid velocity. This feature is also observed when considering the distribution of currents or flow velocity respectively. As shown in Fig. 7 the current distribution decays faster for both structures. However, the distributions appear to be qualitatively similar,

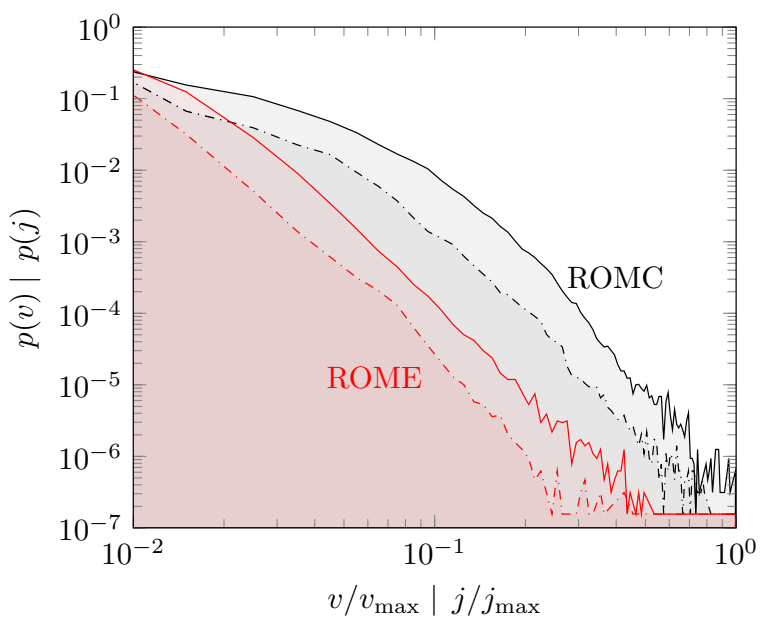

FIG. 7. (Color online) Comparison of velocity magnitude distribution $p(v)$ (solid) and current magnitude distribution $p(j)$ (dashed) in ROMC (black) and ROME (red) structures shown in Fig. 6. Both distributions, i.e., $p(v)$ and $p(j)$, follow similar trends, however, the velocity decays with a significantly faster amplitude. which could explain the surprisingly accurate prediction of the Katz-Thompson conjecture.

\section{TRANSPORT AND MORPHOLOGY}

Transport in Boolean models is either described by percolation theory or by effective medium approximations [22]. In percolation theory one assumes that close to $\phi_{c}$ transport properties are described by power laws and far away from $\phi_{c}$ effective medium theories are applied. In both cases the data agrees qualitatively with this assumption. In Fig. 5 both the experimentally (closed symbols) and numerically obtained values (open symbols) for the permeabilities $k$ are plotted versus several quantities for void and grain percolation. As shown in Fig. 5(a) and Fig. 5(b) the permeabilities vanish close to $\phi_{c}$ and approach the value of an unpatterned channel for $\phi \rightarrow 1$. Due to the finite size of the samples the measured permeabilities scatter more and more as the percolation threshold is approached. Due to the finite system size some structures have porosities below $\phi_{c}$ and are still conductive (technically for these models $\phi_{c}$ is only well defined for infinite systems). This becomes particularly clear when $k$ is plotted vs. the rescaled porosity [see Figs. 5(c), 5(d)], as motivated by Archie's law. The measured permeabilities collapse onto a single curve within the experimental accuracy. However, in the case of negative rescaled porosities, Eq. (4) obviously cannot be applied. In dependence of the open porosity for void percolation, ROMC structures have a higher permeability than ROME structures for equal $\phi_{o}$ [see Fig. 5(e)]. In the case of grain percolation this situation is reversed [see Fig. 5(f)]. This fact can be explained from the morphology of the velocity fields as shown below.

Compared to circles the ellipses form more elongated interconnected obstacles with a significant amount of stagnant parts between grains. This reduces the permeability significantly. In the case of grain percolation the ellipses form 
more direct pathways for the flow, which explains the higher permeability compared to ROMC structures. The experimental and numerical results support that

$$
k=c l_{c}^{2}\left(\frac{1-\chi_{o}}{N}\right)^{\alpha}
$$

for the void percolation models [7] where $\alpha$ is a free parameter. Equation (17) can be justified from the velocity magnitude distributions inside the porous structures shown in Fig. 6. When comparing ROMC and ROME structures it becomes clear that circular grains form more compact obstacles at equal $\phi_{o}$ compared to elliptical grains [compare Figs. 6(e) and Fig. 6(f)], because the probability to overlap and form more tortuous pathways as well as dead ends where no flow occurs is larger for elongated ellipses at equal grain densities. This fact is captured by the factor $\left(1-\chi_{o}\right) / N$, which can be interpreted as the number density of obstacles formed by joined grains. All data points for void percolation are well described by a fit to Eq. (17) with $\alpha_{v}=1.27$ [see Fig. $5(\mathrm{~g})$ ], which is close to the critical exponent $\mu=1.3$ in two dimensions for Archie's law.

One particular shortcoming of Eq. (17) is the explicit dependence on the grain number $N$, which might be unknown or ill-defined for many porous materials, where the formation process is unknown. Also, in the case of grain percolation the use of $N$ in the denominator of Eq. (17) gives even the wrong limit $k=0$ for $N \rightarrow \infty$. Therefore we replace $N$ by an effective grain number $\hat{N}$, which is derived from the morphological correlations of the Minkowski functionals of Boolean models [Eqs. (10)-(12)]. In the continuum one obtains

$$
\hat{N}=\frac{P^{2}}{4 \pi A \phi}-\frac{\chi}{\phi}
$$

and from (13)-(15) on the 2D lattice

$$
\begin{aligned}
\hat{N}= & -L^{2} \ln \left[\frac{\chi}{L^{2} \phi}\left(1-\frac{S}{4 L^{2} \phi}\right)^{-2}\right] \\
& -\left(1-\frac{S}{4 L^{2} \phi}\right)^{-2}+2\left(1-\frac{S}{4 L^{2} \phi}\right)^{-1} .
\end{aligned}
$$

In the case of void percolation $\hat{N}$ is, as expected, typically close to the actual value of $N$ (see Table I). In this case $\hat{N}$ can either be calculated from vectorized images (the binary images of the structures are vectorized using a marching squares algorithm) using Eq. (18) or directly from the raster images using Eq. (19). As shown in Table I, similar values are obtained, with slightly better agreement of $\hat{N}$ and $N$ for the lattice equation. For grain percolation the role of both phases is inverted and $\hat{N}$ does obviously not correspond to $N$, but instead is used to define an effective grain number. As shown in Fig. 5(h) the prediction of the permeability, i.e., the black dashed line, which we got for void percolation, is quite accurate for ROME structures at high porosities but significantly overestimates $k$ for ROMC structures. The reason for this deviation might be the occurrence of many very small isolated obstacles (see Fig. 8) with a size of a few pixels, which significantly influence $\hat{N}$ without strongly influencing $k$. Such isolated obstacles are found more frequently for ROME than for ROMC structures. Looking again at Fig. 5(h), the data
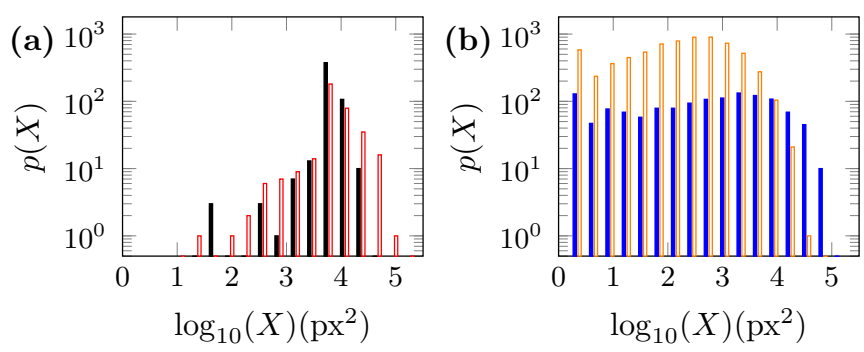

FIG. 8. (Color online) Distribution for obstacle sizes $p(X)$ for (a) void percolation and (b) grain percolation. Open bars correspond to ROME and filled bars correspond to ROMC structures. The histograms for grain percolation show a large number of very small obstacles.

points follow a similar trend as in Fig. 5(g). However, the scattering is significantly stronger. A fit of Eq. (17) to the measured data, which is shown as a green dashed line, yields an exponent $\alpha_{g}=2.05$. The quantitative deviation from $\alpha_{v}$ could indicate that the motivation, which gave rise to Eq. (17), is obviously not directly applicable in the case of $\hat{N}$ for arbitrary structures. The factor $\left(1-\chi_{o}\right) / N$ was interpreted as the number density of obstacles, which are formed by joined individual grains. This interpretation is not that straightforward for grain percolation, since the obstacles are formed by what is left after removing a number $N$ of circular or elliptical areas. However, even in this case the equation does not fail qualitatively, so that we expect a reasonable prediction of $k$ for any structures composed of overlapping grains.

\section{LOW GRAIN DENSITY}

The success of the relation [Eq. (17)] between permeability and Euler characteristic of Boolean models raises the question whether certain results can be obtained analytically or at least semiempirically. In the regime of low grain density analytical results are available for the conductivity $\sigma$ [51]. In this regime, i.e., for $\phi \rightarrow 1$ the Euler characteristic of the conducting phase becomes $\chi_{o} \rightarrow \chi$ as the grain density is so low that individual grains do not overlap. Consequently we can expand Eq. (10)(12) and obtain

$$
-\frac{\chi}{N}=1-\left(1+\frac{\left[4 a E\left(1-\frac{b^{2}}{a^{2}}\right)\right]^{2}}{4 \pi^{2} a b}\right)(1-\phi)+\mathcal{O}\left(\phi^{2}\right),
$$

where $a$ and $b$ are the long and short semiaxis of the ellipse and $E$ is the elliptical integral of the second kind. Here we approximate the circumference of the ellipse $4 a E\left(1-\frac{b^{2}}{a^{2}}\right) \approx$ $\pi \sqrt{2\left(a^{2}+b^{2}\right)}$ and obtain

$$
-\frac{\chi}{N} \approx 1-\frac{(a+b)^{2}}{2 a b}(1-\phi) .
$$

This result is indeed equivalent to the exact result for $\sigma$ of a conductive sheet with a small number of circular obstacles (i.e., the dilute limit) in two dimensions [51] and thus

$$
\frac{\sigma}{\sigma_{0}} \approx-\frac{\chi}{N} \text {. }
$$

For large obstacle densities however, no analytical results are available. In the region close to $\phi_{c}$, where Boolean models 
fall into the universality class of 2D lattice percolation, it is assumed that the conductivity is described by power laws, as previously stated [22]. However, whether the same is true for $\chi_{o}$ is not obvious. In the following we present a numerical analysis of the dependence of $\chi_{o}$ close to $\phi_{c}$, in the intermediate range and far away from $\phi_{c}$ for Boolean models and lattice percolation, in dependence of grain shape and system size, to evaluate the possible universal behavior of $\chi_{o}$ and link the results to percolation theory.

\section{CRITICAL BEHAVIOR}

Close to $\phi_{c}$ transport properties can change dramatically, due to the fractal behavior of the percolating cluster. Results can then depend dramatically on the system size. In percolation theory a universal critical exponent is assumed for the conductivity. Because of the relation between $\phi_{c}$ and $\chi_{o}$ in Eq. (17), the question arises whether $\chi_{o}$ also shows a critical behavior close to $\phi_{c}$. To analyze the dependence of $\chi_{o}$ on the system size and $\phi$ close to $\phi_{c}$ we generate two different types of structures that minimize discretization errors: site percolation on a lattice and ROMR structures in the continuum.

The percolation probability of site percolation systems close to $\phi_{c}$ is described by a universal exponent $\beta=5 / 36$, which is known analytically in two dimensions from conformal field theory (we use the standard notation for critical exponents as common in the literature, e.g., Ref. [26]). Boolean models of randomly overlapping grains fall into the same universality class, so the critical exponents are equal. However, the percolation threshold is nonuniversal and depends on the details of the grains.

In the case of the Euler characteristic the problem is less well understood. Although for many random fields the Euler characteristic can be determined analytically, this is not the case for the Euler characteristic of the open phase, i.e., the percolating cluster. For fractals, the scaling of area, perimeter and Euler characteristic behave differently. While the area scales with one critical exponent, such as in the case of the percolation probability, the perimeter has one additional, and the Euler characteristic has two additional scaling exponents, which are not independent [52,53]. The amplitudes of the scaling relations are in general not known analytically. Therefore, we analyze the scaling behavior of the Euler characteristic numerically.

First, we calculate $\left(1-\chi_{o}\right) / N$ for ROMR structures in dependence of the rescaled porosity. Calculations are performed for system sizes $L=10 a, 20 a, 50 a, 100 a$ where $a$ is the length of the long side of the rectangles. The simulations are repeated for different aspect ratios $1: 1,1: 2,1: 4$ and $1: 10$. An ensemble of 2500 samples for the smallest and six samples for the largest system size was simulated, which, except for the largest system size, results in a negligible error of the mean for all data points. As shown in the resulting curves in Figs. 9(a)-9(d), we observe for all systems two distinct regimes with significantly different $\phi$ dependence. First, a critical regime close to $\phi_{c}$ and second, an effective medium regime where the data points collapse for different aspect ratios, but a significantly different slope of the curve compared to the critical regime is observed. No qualitative change in this behavior is observed for different system sizes. However, due to the rather limited system size of the ROMR structures, it is not obvious whether these two regimes persist for infinite system size.

Therefore, we also simulate site percolation on a 2D square lattice, for which a linear system size of $L=2^{14}$ lattice sites can be achieved with an ensemble of $2^{6}$ realizations and up to $2^{16}$ for the smallest system size $L=2^{5}$. Here, $N$ must be replaced by $(1-p) L^{2}$, which, $p$ being the probability that
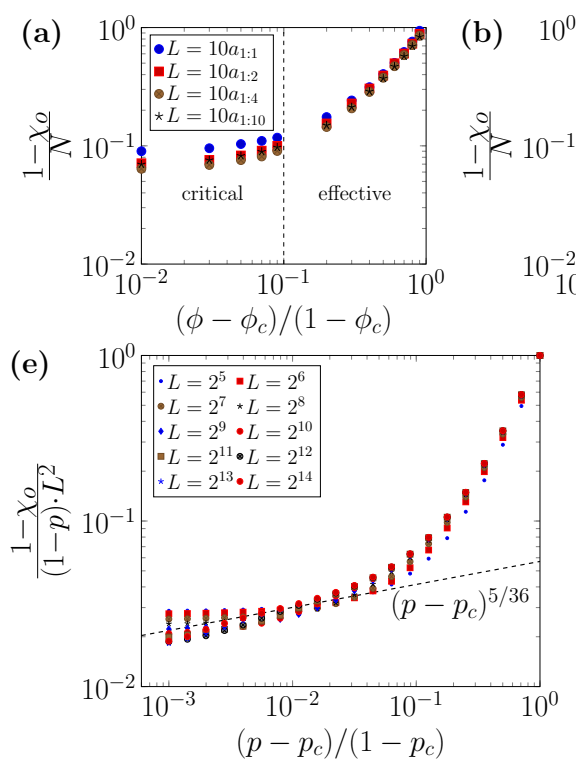
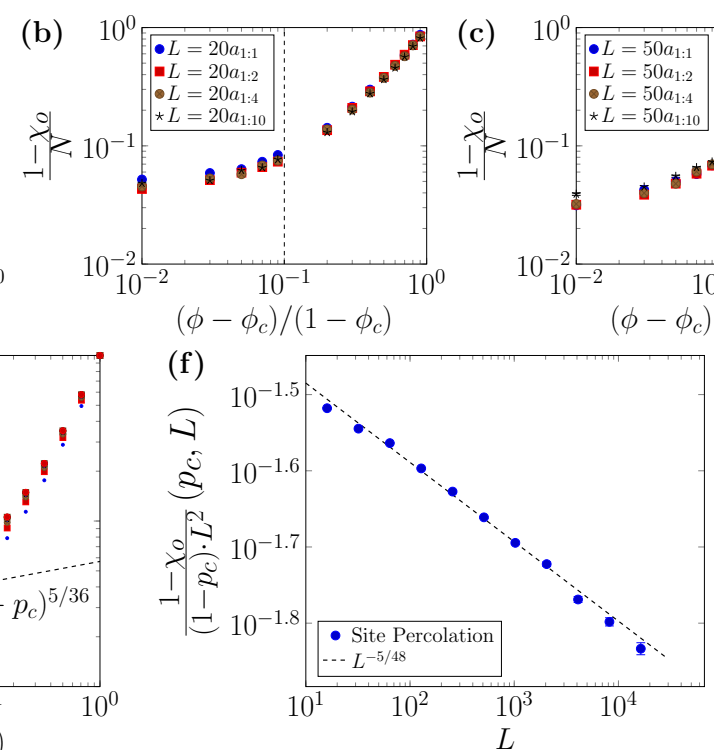
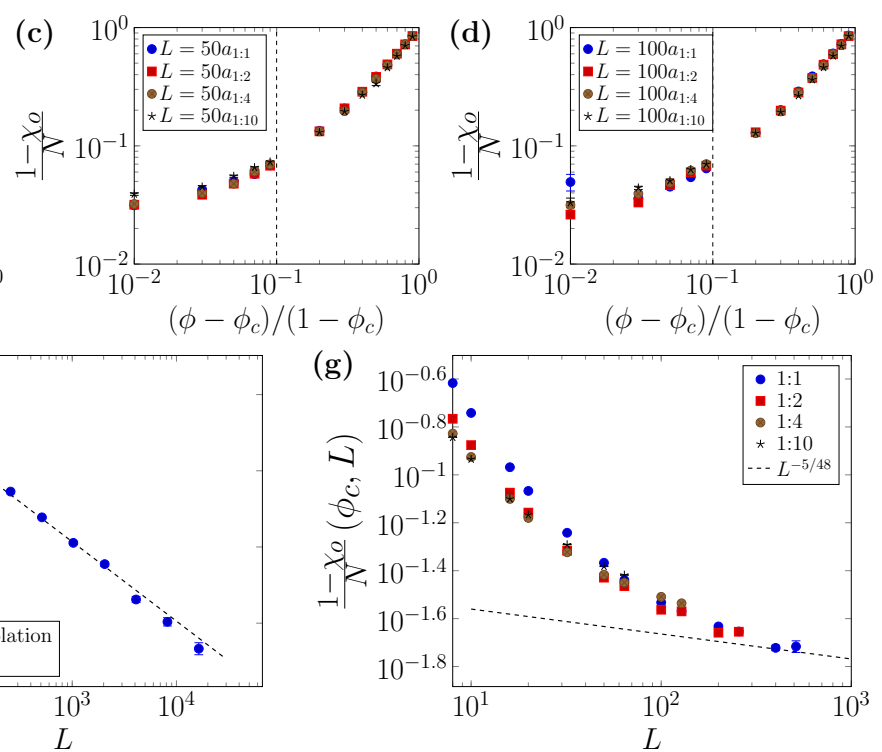

FIG. 9. (Color online) Behavior of the Euler characteristic of the percolating cluster $\chi_{o}$ for (a)-(d) void percolation of different ROMR systems (aspect ratios $1: 1,1: 2,1: 4,1: 10$ ) as a function of the porosity $\phi$ and (e) site percolation on a 2D lattice function of the occupation probability $p$. For the ROMR structures system sizes of $L=10 a, 20 a, 50 a$, and $100 a$ have been simulated. For site percolation $L=2^{2}, \ldots, 2^{14}$. (f) Finite-size scaling analysis of $\chi_{o}$ for site percolation at the percolation threshold for difference linear system size $L$. (g) Finite-size scaling of $\chi_{o}$ in dependence of the linear system size $L$ for ROMR structures. 
a site is conducting, corresponds to the volume occupied by obstacles. In this system, as shown in Fig. 9(e), we find exactly the same behavior as for ROMR structures, for all system sizes. This further supports our assumption that the occurrence of two scaling regimes persists for other grain shapes and larger system sizes.

However, a direct determination of the scaling exponent from finite-size system simulations is not feasible [26]. Instead, an established method to extract critical exponents numerically is used from finite-size scaling [25,26]. Here, we only give a brief description of the method: If we combine Eq. (4) and Eq. (17), we get $\left(1-\chi_{o}\right) / N \propto\left(\phi-\phi_{c}\right)^{\beta}$, where $\beta=\mu / \alpha$. Because the correlation length $\xi$ diverges at $\phi_{c}$ according to a power law, we can use that $\xi \propto\left(\phi-\phi_{c}\right)^{-v}$ to obtain $\left(1-\chi_{o}\right) / N \propto \xi^{-\beta / v}$. Since $\xi$ is infinite at $\phi=\phi_{c}, L$ becomes the confining length scale of the system and we obtain (1 $\left.\chi_{o}\right) / N \propto L^{-\beta / \nu}$. This assumption is now tested numerically. Here, our results might differ for site percolation [Fig. 9(e)] and ROMR structures [Fig. 9(f)]. For site percolation we clearly observe a power law with an exponent of $-\beta / \nu=-5 / 48$ in perfect agreement with percolation theory. This suggests that $\beta=5 / 36$ indeed describes the scaling of the open Euler characteristic. However, for ROMR structures the slope of the curve is significantly different for small $L$ and only at large $L$ becomes consistent with a scaling of $L^{-\beta / v}$ (see dashed line). Apparently the ROMR system is more sensitive to finite-size effects. However, larger $L$ are computationally too expensive, so that $L<256$ for square grains and worse for larger aspect ratios, since the amount of grains at a fixed $\phi$ diverges with the aspect ratio.

Nevertheless, our numerical results support the following interpretation: When we connect the scaling of the open Euler characteristic to the experimental observation the critical exponent seems to be irrelevant with respect to our experimental system sizes. In the critical regime from Eq. (4) percolation theory would yield

$$
k \propto\left(\frac{1-\chi_{o}}{N}\right)^{\mu / \beta},
$$

which we obviously do not observe in the experiment. Instead our interpretation is that the power law from Eq. (17) is related to the effective regime. Since in this regime the open Euler characteristic collapses, we argue that the same should be true for conductivity and permeability, however with a significantly different exponent (compared to $\beta$ ) with a value close to 1 . A more detailed analysis of this problem, however, requires either new simulations or experimental studies of conductivity or permeability of structures with much larger system size. Consequently, a significant improvement of the computational and/or experimental effort is required. So far, only 20 structures could be measured and simulated. For a higher number of samples a reliable automation of the experiment would be required. For large structures close to $\phi_{c}$ the resolution of the experiment must be improved by at least one order of magnitude. The same is true for the computational time required to solve the conductivity or permeability problem.

\section{SUMMARY}

We have analyzed the permeability and conductivity of porous micromodels composed of randomly overlapping grains (Boolean models). We have analyzed void and grain percolation for overlapping circles and ellipses (i.e., structures where the void is conductive and structures where the grains are conductive). In all cases the relation between permeability and conductivity is well predicted by the Katz-Thompson model. In the case of void percolation the permeability can be deduced from the Euler characteristic of the percolating cluster normalized to the total number of grains, which required $a$ priori knowledge of the grain density. For grain percolation a similar approach is studied based on the definition of an effective grain number $\hat{N}$, which is calculated from the global Minkowski functionals of the structures. This approach works qualitatively for ROME structures, but overestimates $k$ for ROMC structures, due to the sensitivity of $\hat{N}$ on the occurrence of isolated pixels, which otherwise do not strongly affect $k$. For void percolation in the low grain-density limit it can be analytically shown that the formation factor is given by the Euler characteristic. The critical behavior of the Euler characteristic of the percolation cluster $\chi_{o}$ for $\phi \rightarrow \phi_{c}$ is analyzed numerically to link our results to percolation theory. For the 2D square lattice, we find that $\chi_{o}$ scales with the critical exponent $\beta$ only very close to $\phi_{c}$. Further away from $\phi_{c}$, an effective regime is found for both square lattice and Boolean models where the values of $\chi_{o}$ overlap for different systems, i.e., different grain shapes and system sizes, justifying the applicability of our model to many different types of structures.

A remaining question is the applicability to fully threedimensional (3D) porous media. In principle, 3D models are accessible experimentally, e.g., via 3D printing, and have also been studied numerically [54]. Also MFs are well understood in the $3 \mathrm{D}$ case, e.g., the Euler characteristic also vanishes close to $\phi_{c}$. Therefore, it is reasonable to assume that Eq. (17) could hold in three dimensions, even though there is no intuitive interpretation similar to the $2 \mathrm{D}$ case. It must be expected however, that measurements and simulations are significantly more challenging and computationally more expensive.

\section{ACKNOWLEDGMENTS}

We thank Jan Götz for the support on LB simulations for inverted Boolean structures. We also acknowledge funding by the German Science Foundation (DFG) through Grants No. ME1361/12 and No. SCHR-1148/3.
[1] Committee on Chemical Engineering Frontiers: Research Needs and Opportunities, National Research Council, Frontiers in Chemical Engineering: Research Needs and Opportunities (The National Academies Press, Washington, D.C., 1988).

[2] M. Sahimi, Rev. Mod. Phys. 65, 1393 (1993).
[3] J. Bear, Dynamics of Fluids in Porous Media (Dover Publications, Mineola, 2013).

[4] S. Torquato, Appl. Mech. Rev. 44, 37 (1991).

[5] C. H. Arns, M. A. Knackstedt, and K. R. Mecke, Phys. Rev. Lett. 91, 215506 (2003). 
[6] K. Mecke and C. H. Arns, J. Phys.: Condens. Matter 17, S503 (2005).

[7] C. Scholz, F. Wirner, J. Götz, U. Rüde, G. E. Schröder-Turk, K. Mecke, and C. Bechinger, Phys. Rev. Lett. 109, 264504 (2012).

[8] P. Lehmann, M. Berchtold, B. Ahrenholz, J. Tölke, A. Kästner, M. Krafczyk, H. Flühler, and H. Künsch, Adv. Water Resour. 31, 1188 (2008).

[9] S. Yang, M. Liang, B. Yu, and M. Zou, Microfluid. Nanofluid. 18, 1085 (2015).

[10] J. Kozeny, Sitzber. Akad. Wiss. Wien, Math-naturw 136, Abt. II a, p. 271 (1927).

[11] A. J. Katz and A. H. Thompson, Phys. Rev. B 34, 8179 (1986).

[12] P. N. Sen, J. N. Roberts, and B. I. Halperin, Phys. Rev. B 32, 3306 (1985).

[13] A. Bunde, H. Harder, and S. Havlin, Phys. Rev. B 34, 3540 (1986).

[14] M. Octavio, A. Octavio, J. Aponte, R. Medina, and C. J. Lobb, Phys. Rev. B 37, 9292 (1988).

[15] N. Martys and E. J. Garboczi, Phys. Rev. B 46, 6080 (1992).

[16] C. H. Arns, M. A. Knackstedt, and N. S. Martys, Phys. Rev. E 72, 046304 (2005)

[17] J. R. Quispe, R. E. Rozas, and P. G. Toledo, Chem. Eng. J. 111, 225 (2005).

[18] L. Andersson, A. C. Jones, M. A. Knackstedt, and L. Bergström, Acta Mater. 59, 1239 (2011).

[19] Y.-B. Yi and A. M. Sastry, Phys. Rev. E 66, 066130 (2002).

[20] J. A. Quintanilla and R. M. Ziff, Phys. Rev. E 76, 051115 (2007).

[21] S. Kirkpatrick, Rev. Mod. Phys. 45, 574 (1973).

[22] M. Sahimi, B. D. Hughes, L. E. Scriven, and H. T. Davis, J. Phys. C 16, L521 (1983).

[23] J. Tobochnik, D. Laing, and G. Wilson, Phys. Rev. A 41, 3052 (1990).

[24] P. Grassberger, Phys. A (Amsterdam, Neth.) 262, 251 (1999).

[25] D. Stauffer and A. Aharony, Introduction to percolation theory (CRC Press, Boca Raton, 1994).

[26] K. Christensen and N. R. Moloney, Complexity and criticality, Vol. 1 (Imperial College Press, London, 2005).

[27] S. R. Quake and A. Scherer, Science 290, 1536 (2000).

[28] G. Whitesides and A. Stroock, Phys. Today 54(6), 42 (2001).

[29] J. C. Crocker and D. G. Grier, J. Colloid Interface Sci. 179, 298 (1996).

[30] J. G. Santiago, S. T. Wereley, C. D. Meinhart, D. J. Beebe, and R. J. Adrian, Exp. Fluids 25, 316 (1998).

[31] R. Lindken, M. Rossi, S. Große, and J. Westerweel, Lab Chip 9 , 2551 (2009).
[32] M. Auset and A. A. Keller, Water Resour. Res. 40, W03503 (2004).

[33] M. Auset and A. A. Keller, Water Resour. Res. 42, W12S02 (2006).

[34] C. Scholz, F. Wirner, Y. Li, and C. Bechinger, Exp. Fluids 53, 1327 (2012).

[35] D. Frenkel, Phys. A (Amsterdam, Neth.) 313, 1 (2002).

[36] M. E. Staben, A. Z. Zinchenko, and R. H. Davis, Phys. Fluids 15, 1711 (2003).

[37] C. Pozrikidis, J. Fluid Mech. 261, 199 (1994).

[38] H. Bruus, Theoretical Microfluidics (Oxford University Press, Oxford, 2007).

[39] S. N. Chiu, D. Stoyan, W. S. Kendall, and J. Mecke, Stochastic geometry and its applications (Wiley, New York, 2013).

[40] M. Spanner, S. K. Schnyder, F. Höfling, T. Voigtmann, and T. Franosch, Soft Matter 9, 1604 (2013).

[41] S. K. Schnyder, M. Spanner, F. Höfling, T. Franosch, and J. Horbach, Soft Matter 11, 701 (2015).

[42] K. Mecke and H. Wagner, J. Stat. Phys. 64, 843 (1991).

[43] K. Mecke, T. Buchert, and H. Wagner, Astron. Astrophys. 288, 697 (1994).

[44] D. A. Klain and G.-C. Rota, Introduction to geometric probability, 1st ed. (Cambridge University Press, Cambridge, 1997), pp. XIV, $178 \mathrm{~S}$.

[45] H. Mantz, K. Jacobs, and K. Mecke, J. Stat. Mech.: Theory Exp. (2008) P12015.

[46] G. E. Schröder-Turk, W. Mickel, S. C. Kapfer, M. A. Klatt, F. M. Schaller, M. J. F. Hoffmann, N. Kleppmann, P. Armstrong, A. Inayat, D. Hug et al., Adv. Mater. 23, 2535 (2011).

[47] G. E. Schröder-Turk, W. Mickel, S. C. Kapfer, F. M. Schaller, B. Breidenbach, D. Hug, and K. Mecke, New J. Phys. 15, 083028 (2013).

[48] M. Hilpert and C. T. Miller, Adv. Water Resour. 24, 243 (2001).

[49] W. Mickel, S. Münster, L. M. Jawerth, D. A. Vader, D. A. Weitz, A. P. Sheppard, K. Mecke, B. Fabry, and G. Schröder-Turk, Biophys. J. 95, 6072 (2008).

[50] J. S. Andrade, Jr., M. P. Almeida, J. Mendes Filho, S. Havlin, B. Suki, and H. E. Stanley, Phys. Rev. Lett. 79, 3901 (1997).

[51] W. Xia and M. F. Thorpe, Phys. Rev. A 38, 2650 (1988).

[52] K. R. Mecke, in Statistical Physics and Spatial Statistics (Springer, Berlin, 2000), pp. 111-184.

[53] P. Schönhöfer and K. Mecke, in Fractal Geometry and Stochastics $V$ (Springer, Berlin, 2015), pp. 39-52.

[54] G. G. Pereira, P. M. Dupuy, P. W. Cleary, and G. W. Delaney, Prog. Comput. Fluid Dy. 12, 176 (2012). 